\title{
QUASISYMMETRIC EMBEDDING OF THE INTEGER SET AND ITS QUASICONFORMAL EXTENSION
}

\author{
Hiroki Fujino \\ Nagoya University, Graduate School of Mathematics \\ Furo-cho Chikusa-ku Nagoya 464-8602, Japan; m12040w@math.nagoya-u.ac.jp
}

\begin{abstract}
We prove that an injection from the integer set into the real line admits a quasiconformal extension to the complex plane if and only if it is quasisymmetric.
\end{abstract}

\section{Introduction}

Let $E \subset \mathbf{C}$ be a discrete subset. In [3], to study the Teichmüller space of the punctured plane $\mathbf{C} \backslash \mathbf{Z}$, the author gave some criteria for $\mathbf{C} \backslash E$ to be quasiconformally equivalent to $\mathbf{C} \backslash \mathbf{Z}$ (that is, there exists a quasiconformal mapping $F: \mathbf{C} \rightarrow \mathbf{C}$ such that $F(E)=\mathbf{Z}$ ). In this paper, furthermore, we investigate the correspondences between $E$ and $\mathbf{Z}$ which are the restrictions of global quasiconformal mappings $F: \mathbf{C} \rightarrow \mathbf{C}$ such that $F(E)=\mathbf{Z}$. A motivation of this attempt is to study the Teichmüller modular group of $\mathbf{C} \backslash \mathbf{Z}$ and its action.

Let $\eta:[0, \infty) \rightarrow[0, \infty)$ be a homeomorphism and $f: X \rightarrow \mathbf{R}^{n}$ be an $\eta$-quasisymmetric embedding from a subset $X \subset \mathbf{R}^{n}$ into $\mathbf{R}^{n}$. The theory of quasisymmetry and its quasiconformal extension originated from the well known study for $n=1$ and $X=\mathbf{R}$ by Beurling-Ahlfors [2]. They proved that a homeomorphism $f: \mathbf{R} \rightarrow \mathbf{R}$ admits a quasiconformal extension $F: \mathbf{C} \rightarrow \mathbf{C}$ if and only if $f$ is quasisymmetric. This result enables us to treat the universal Teichmüller space, the Teichmüller space of the unit disk, as the space of all orientation preserving quasisymmetric homeomorphisms of the unit circle which fix given three points. Later, Väisälä posed the following question in [7, Question 8] which is still open: Can quasisymmetric embed$\operatorname{ding} f: X \rightarrow \mathbf{R}^{n}$ be extended to a $K$-quasiconformal mapping $F: \mathbf{R}^{2 n} \rightarrow \mathbf{R}^{2 n}$ with a constant $K=K(n, \eta) \geq 1$ which depends only on $n$ and $\eta$ ?

For example, Alestalo-Väisälä showed that if $f: X \rightarrow \mathbf{R}^{n}$ is $M$-biLipschitz, then there always exists a $\sqrt{7} M^{2}$-biLipschitz extension $F: \mathbf{R}^{2 n} \rightarrow \mathbf{R}^{2 n}$ of $f$, see [1, Theorem 5.5]. On the other hand, for quasisymmetric embeddings, there is an obstacle; Trotsenko-Väisälä proved in [5, Theorem 6.6] that if $X \subset \mathbf{R}^{n}$ is not relatively connected, then there exists a quasisymmetric embedding $f: X \rightarrow \mathbf{R}^{n}$ which cannot be extended to a quasisymmetric embedding $F: \mathbf{R}^{n} \rightarrow \mathbf{R}^{N}$ for any $N \geq n$. Since global quasiconformal mappings $F: \mathbf{R}^{2 n} \rightarrow \mathbf{R}^{2 n}$ are also quasisymmetric (see [4, Theorem 11.14]), this fact implies that the Väisälä problem cannot be solved affirmatively for general subsets $X$ even if $n=1$.

According to the recent study by Vellis [8], he showed that if $X \subset \mathbf{R}$ is $M$ relatively connected, then every $\eta$-quasisymmetric embedding $f: X \rightarrow \mathbf{R}^{n}$ can be extended to an $\eta^{\prime}$-quasisymmetric embedding $F: \mathbf{R} \rightarrow \mathbf{R}^{N}$, where $\eta^{\prime}$ depends only on $\eta$ and $M$, and $N(\geq n)$ depends only on $n, \eta$, and $M$. Considering the one

https://doi.org/10.5186/aasfm.2017.4230

2010 Mathematics Subject Classification: Primary 51M04; Secondary 51M05.

Key words: Quasiconformal mapping, quasisymmetric mapping. 
dimensional case of the Väisälä problem, it is interesting to find out whether we can choose $N=2$ uniformly when $n=1$ in the Vellis' result.

Let us consider the case of $n=1$ and $X=\mathbf{Z}$. In this paper, we would like to give detailed observations on quasisymmetric embeddings $f: \mathbf{Z} \rightarrow \mathbf{R}$, as an example of a relatively connected set for which the Väisälä problem can be solved affirmatively;

Theorem A. (Extendability of quasisymmetric embeddings of $\mathbf{Z}$ ) Every $\eta$-quasisymmetric embedding $f: \mathbf{Z} \rightarrow \mathbf{R}$ admits a $K=K(\eta)$-quasiconformal extension $F: \mathbf{C} \rightarrow \mathbf{C}$ where $K(\eta)$ is a constant depending only on $\eta$.

Recall that, in the study of Beurling-Ahlfors, they considered quasisymmetric homeomorphisms of the real line. Although homeomorphisms of the real line are monotone, quasisymmetric embedding $f: \mathbf{Z} \rightarrow \mathbf{R}$ need not to be monotone. This is one of the difficulty in our study.

To prove Theorem A, first, we will discuss the extendability of quasisymmetric automorphisms $f: \mathbf{Z} \rightarrow \mathbf{Z}$ in Section 4.1.

Theorem B. (Extendability of quasisymmetric automorphisms of Z) For a bijection $f: \mathbf{Z} \rightarrow \mathbf{Z}$, the following conditions are quantitatively equivalent;

1. $f$ is $\eta$-quasisymmetric.

2. $f$ satisfies the $\lambda$-three point condition.

3. $f$ is $M$-biLipschitz.

4. $f$ admits an $M$-biLipschitz extension $F: \mathbf{C} \rightarrow \mathbf{C}$.

5. $f$ admits a $K$-quasiconformal extension $F: \mathbf{C} \rightarrow \mathbf{C}$.

The $\lambda$-three point condition is defined as follows.

Definition 1.1. Let $A \subset \mathbf{R}$ be a subset and let $\lambda \geq 1$. Then we say that an injection $f: A \rightarrow \mathbf{R}$ satisfies the $\lambda$-three point condition if the following inequality holds for any $x, y, z \in A$ with $x<y \leq z$;

$$
\left|\frac{f(x)-f(y)}{f(x)-f(z)}\right| \leq \lambda
$$

Note that the three point condition does not depend on the distances of $x, y, z \in$ $A$. Moreover, if $f: A \rightarrow \mathbf{R}$ satisfies the $\lambda$-three point condition, and if $h: A \rightarrow \mathbf{R}$ is strictly monotone increasing, then $f \circ h^{-1}: h(A) \rightarrow \mathbf{R}$ also satisfies the $\lambda$-three point condition.

We emphasise that Theorem B does not only state that every quasisymmetric automorphism of $\mathbf{Z}$ is quasiconformally extendable, but also characterizes quasisymmetry by a simple condition, the three point condition. Further, an analogous theorem holds for quasisymmetric automorphisms of $E=\left\{e^{n}\right\}_{n \in \mathbf{Z}}$, see Section 4.2.

Next, we will consider a subset of $\mathbf{R}$ which is an image of a quasisymmetric embedding $f: \mathbf{Z} \rightarrow \mathbf{R}$ in Section 5, to complete the proof of Theorem A. In this case, such subsets can also be characterized by a simple geometric condition as follows;

Theorem C. (Characterization of quasisymmetric images) For a subset $E \subset \mathbf{R}$, the following conditions are quantitatively equivalent;

1. There exists an $\eta$-quasisymmetric bijection $f: \mathbf{Z} \rightarrow E$.

2. $E$ can be written as a monotone increasing sequence $E=\left\{a_{n}\right\}_{n \in \mathbf{Z}}$ with $a_{n} \rightarrow \pm \infty$ as $n \rightarrow \pm \infty$, and there exists a constant $M \geq 1$ such that the following inequality holds for all $n \in \mathbf{Z}$ and $k \in \mathbf{N}$;

$$
\frac{1}{M} \leq \frac{a_{n+k}-a_{n}}{a_{n}-a_{n-k}} \leq M .
$$


3. There exists a $K$-quasiconformal mapping $F: \mathbf{C} \rightarrow \mathbf{C}$, such that $F(\mathbf{Z})=E$.

\section{Definitions and basic properties}

First, let $\eta:[0, \infty) \rightarrow[0, \infty)$ be a homeomorphism and $X \subset \mathbf{C}$ be a subset. An injection $f: X \rightarrow \mathbf{C}$ is said to be $\eta$-quasisymmetric if the following inequality holds for any three points $x, y, z \in X(x \neq z)$;

$$
\left|\frac{f(x)-f(y)}{f(x)-f(z)}\right| \leq \eta\left(\left|\frac{x-y}{x-z}\right|\right) .
$$

If $x \neq y$, replacing $y$ and $z$, the following lower estimate also holds;

$$
\left|\frac{f(x)-f(y)}{f(x)-f(z)}\right| \geq \eta\left(\left|\frac{x-y}{x-z}\right|^{-1}\right)^{-1} .
$$

Notice that if there exists at least one $\eta$-quasisymmetric mapping (and $X$ contains at least two elements), applying (QS) to $y=z$, it turns out that $\eta$ must satisfy $\eta(1) \geq 1$. Further if $X \subset \mathbf{R}$ and $f(X) \subset \mathbf{R}$, then

$$
\left|\frac{f(x)-f(y)}{f(x)-f(z)}\right| \leq \eta\left(\left|\frac{x-y}{x-z}\right|\right) \leq \eta(1)
$$

for all $x, y, z \in X$ with $x<y \leq z$, that is, $f$ satisfies the $\eta(1)$-three point condition, see Definition 1.1.

Next, let $K \geq 1$ and $\Omega \subset \mathbf{C}$ be a domain. An homeomorphism $f: \Omega \rightarrow \mathbf{C}$ into $\mathbf{C}$ is said to be $K$-quasiconformal if the following inequality holds for all curve families $\mathscr{F}$ in $\Omega$;

$$
\frac{1}{K} \bmod \mathscr{F} \leq \bmod f(\mathscr{F}) \leq K \bmod \mathscr{F}
$$

Here, for a curve family $\mathscr{F}$ in $\Omega, \bmod \mathscr{F}$ is defined by

$$
\bmod \mathscr{F}=\inf \int_{\Omega} \rho^{2}(x+i y) d x d y
$$

where the infimum is taken over all non-negative Borel measurable functions $\rho: \Omega \rightarrow$ $[0, \infty]$ which satisfy

$$
\int_{\gamma} \rho(z)|d z| \geq 1
$$

for all rectifiable $\gamma \in \mathscr{F}$.

These two concepts are closely related by the so-called egg-yolk principle, see $[4$, Theorem 11.14]. In particular, for homeomorphisms from $\mathbf{C}$ onto itself, the quasiconformality and the quasisymmetry are quantitatively equivalent.

Finally, let $X \subset \mathbf{C}$ be a subset and $M \geq 1$. Then a mapping $f: X \rightarrow \mathbf{C}$ is said to be $M$-biLipschitz if

$$
\frac{1}{M}|x-y| \leq|f(x)-f(y)| \leq M|x-y|
$$

for any $x, y \in X$. If $f: X \rightarrow \mathbf{C}$ is $M$-biLipschitz, then

$$
\left|\frac{f(x)-f(y)}{f(x)-f(z)}\right| \leq M^{2}\left|\frac{x-y}{x-z}\right|
$$

for all $x, y, z \in X(x \neq z)$. Thus every $M$-biLipschitz mappping is $\eta(t)=M^{2} t$ quasisymmetric. 


\section{Preliminary lemma}

The following lemma will be used to prove both Theorem B and Theorem C.

Lemma 3.1. Let $E=\left\{a_{n}\right\}_{n \in \mathbf{Z}} \subset \mathbf{R}$ be a monotone increasing sequence. If a bijection $g: E \rightarrow \mathbf{Z}$ satisfies the $\mu$-three point condition $(\mu \geq 1)$, then for any $n \in \mathbf{Z}$ and $k \in \mathbf{N}$, the following inequality holds;

$$
\frac{k}{2 \mu}<\left|g\left(a_{n}\right)-g\left(a_{n+k}\right)\right|<2 \mu k .
$$

Proof. We first prove the following estimation;

Claim 1. $\left|g\left(a_{n}\right)-g\left(a_{n+1}\right)\right|<2 \mu$ for any $n \in \mathbf{Z}$.

Proof. Since $\mu \geq 1$, it suffices to consider the case where $\left|g\left(a_{n}\right)-g\left(a_{n+1}\right)\right| \geq 2$. Then we may assume $g\left(a_{n+1}\right)>g\left(a_{n}\right)$ since the same argument mentioned below can be applied to the case where $g\left(a_{n}\right)>g\left(a_{n+1}\right)$.

Letting $m \leq n$ satisfy

$$
g\left(a_{m}\right)=\max \left\{g\left(a_{j}\right) \mid j \leq n \text { and } g\left(a_{n}\right) \leq g\left(a_{j}\right)<g\left(a_{n+1}\right)\right\}
$$

and $\ell \in \mathbf{Z}$ satisfy $g\left(a_{\ell}\right)=g\left(a_{m}\right)+1$ ( then $\ell \geq n+1$ by the construction), we can construct $m, \ell \in \mathbf{Z}$ which satisfy the following conditions, see Figure 1;

1. $m \leq n$ and $n+1 \leq \ell$,

2. $g\left(a_{n}\right) \leq g\left(a_{m}\right)<g\left(a_{\ell}\right)=g\left(a_{m}\right)+1 \leq g\left(a_{n+1}\right)$.

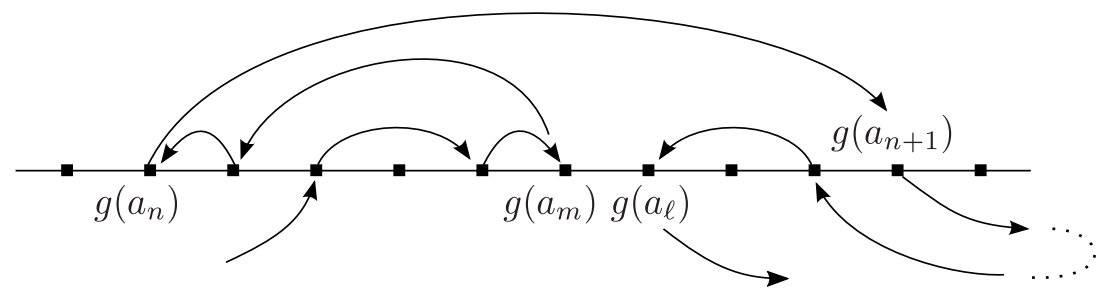

Figure 1. A part of the orbit of the sequence $\left(g\left(a_{j}\right)\right)_{j \in \mathbf{Z}}$.

First, suppose $g\left(a_{m}\right)-g\left(a_{n}\right) \geq\left(g\left(a_{n+1}\right)-g\left(a_{n}\right)\right) / 2 \geq 1$. By the three point condition,

$$
\mu \geq\left|\frac{g\left(a_{m}\right)-g\left(a_{n}\right)}{g\left(a_{m}\right)-g\left(a_{\ell}\right)}\right|=g\left(a_{m}\right)-g\left(a_{n}\right) \geq \frac{g\left(a_{n+1}\right)-g\left(a_{n}\right)}{2} .
$$

Thus we have $g\left(a_{n+1}\right)-g\left(a_{n}\right)<2 \mu$.

Next, suppose $g\left(a_{m}\right)-g\left(a_{n}\right)<\left(g\left(a_{n+1}\right)-g\left(a_{n}\right)\right) / 2$. Then $g\left(a_{n+1}\right)-g\left(a_{m}\right)>$ $\left(g\left(a_{n+1}\right)-g\left(a_{n}\right)\right) / 2$. Similarly we have $g\left(a_{n+1}\right)-g\left(a_{n}\right)<2 \mu$.

Claim 2. Lemma 3.1 holds.

Proof. (Upper bound) By the triangle inequality, it immediately follows from Claim 1 that $\left|g\left(a_{n}\right)-g\left(a_{n+k}\right)\right|<2 \mu k$.

(Lower bound) Since the open interval

$$
\left(g\left(a_{n}\right)-\frac{k}{2}, g\left(a_{n}\right)+\frac{k}{2}\right)
$$

contains at most $k-1$ integers except $g\left(a_{n}\right)$, there exists an integer $m \in \mathbf{Z}(n<m \leq$ $n+k)$ such that

$$
\left|g\left(a_{n}\right)-g\left(a_{m}\right)\right| \geq \frac{k}{2}
$$


By the three point condition, we obtain

$$
\mu \geq\left|\frac{g\left(a_{n}\right)-g\left(a_{m}\right)}{g\left(a_{n}\right)-g\left(a_{n+k}\right)}\right| \geq \frac{k}{2\left|g\left(a_{n}\right)-g\left(a_{n+k}\right)\right|},
$$

that is, $\left|g\left(a_{n}\right)-g\left(a_{n+k}\right)\right|>k / 2 \mu$.

\section{Extendability of quasisymmetric automorphisms of $\mathrm{Z}$}

The aim of this section is to prove Theorem B. After proving Theorem B, we also discuss the extendability of quasisymmetric automorphisms of $E=\left\{e^{n}\right\}_{n \in \mathbf{Z}}$.

\subsection{Proof of Theorem B.}

Theorem B. (Extendability of quasisymmetric automorphisms of Z) For a bijection $f: \mathbf{Z} \rightarrow \mathbf{Z}$, the following conditions are quantitatively equivalent;

1. $f$ is $\eta$-quasisymmetric.

2. $f$ satisfies the $\lambda$-three point condition.

3. $f$ is $M$-biLipschitz.

4. $f$ admits an $M$-biLipschitz extension $F: \mathbf{C} \rightarrow \mathbf{C}$.

5. $f$ admits a $K$-quasiconformal extension $F: \mathbf{C} \rightarrow \mathbf{C}$.

Proof. First, $(1) \Rightarrow(2)$ is clear, see Section 2.

Next, we suppose $f$ satisfies the $\lambda$-three point condition. By applying Lemma 3.1 to $f$ and $E=\mathbf{Z}$ (that is, $a_{n}=n$ ), we have

$$
\frac{k}{2 \lambda}<|f(n+k)-f(n)|<2 \lambda k,
$$

for all $n \in \mathbf{Z}$ and $k \in \mathbf{N}$. Thus we obtain $(2) \Rightarrow(3)$.

$(3) \Rightarrow(4)$ is already proved by Alestalo-Väisälä [1, Theorem 5.5].

Generally, $M$-biLipschitz mappings are $\eta(t)=M^{2} t$-quasisymmetric. Further, $\eta$ quasisymmetric mappings defined on connected open subsets are $K$-quasiconformal, where $K$ is a constant depending only on $\eta$. Thus we have $(4) \Rightarrow(5)$.

Finally, $(5) \Rightarrow(1)$ is also clear, since $K$-quasiconformal self-homeomorphisms of $\mathbf{C}$ are $\eta$-quasisymmetric with an $\eta$ depending only on $K$ (thus the restrictions to $\mathbf{Z}$ are also $\eta$-quasisymmetric with the same $\eta$ ).

4.2. Extendability of quasisymmetric automorphisms of $\left\{e^{n}\right\}_{n \in Z}$. Let $E=\left\{e^{n}\right\}_{n \in \mathbf{Z}}$. We first prove the following.

Proposition 4.1. If a bijection $f: E \rightarrow E$ satisfies $\lambda$-three point condition $(\lambda \geq 1), f(1)=1$, and $f\left(e^{n}\right) \rightarrow 0$ as $n \rightarrow-\infty$, then $f$ is $M_{\lambda}$-biLipschitz, where $M_{\lambda}=\frac{e^{2}}{e-1} \lambda(\lambda+1)^{2}$.

Proof. Let $g:=\log \circ f \circ \exp$, that is, $f\left(e^{n}\right)=e^{g(n)}$ for all $n \in \mathbf{Z}$. Then, by the assumptions, $g(0)=0$ and $g(n) \rightarrow-\infty$ as $n \rightarrow-\infty$. Let $C_{\lambda}=\log (\lambda+1)$.

Claim 1. $g(k)-g(\ell) \leq C_{\lambda}$ if $k<\ell$.

Proof. Since $C_{\lambda}>0$, it suffices to consider the case where $g(k)>g(\ell)$ and $k<\ell$. Since $g(n) \rightarrow-\infty$ as $n \rightarrow-\infty$, there exists an integer $j<k$ such that $g(j)<g(\ell)$. Thus, by the three point condition,

$$
\lambda \geq\left|\frac{e^{g(j)}-e^{g(k)}}{e^{g(j)}-e^{g(\ell)}}\right|=\frac{e^{g(k)-g(\ell)}-e^{-(g(\ell)-g(j))}}{1-e^{-(g(\ell)-g(j))}} .
$$


Since $0<e^{-(g(\ell)-g(j))}<1$, we have $\lambda \geq e^{g(k)-g(\ell)}-1$. Therefore $g(k)-g(\ell) \leq$ $\log (\lambda+1)=C_{\lambda}$.

Claim 2. $-\left(2 C_{\lambda}+1\right) \leq g(n)-n \leq 2 C_{\lambda}+1$ for all $n \in \mathbf{Z}$.

Proof. Let $n>0$. By Claim 1 and $g(0)=0$, we have $g(m) \geq-C_{\lambda}$ for all $m>0$. Thus there exists an integer $j(0 \leq j<n)$ such that $g(j) \geq-C_{\lambda}+n-1$. Using Claim 1 again, we have

$$
C_{\lambda} \geq g(j)-g(n) \geq-C_{\lambda}+n-1-g(n) .
$$

Thus $g(n)-n \geq-\left(2 C_{\lambda}+1\right)$. Further, to obtain a contradiction, we suppose $g(n)-n>$ $2 C_{\lambda}+1$. Note that $g(n)>0$. Let

$$
\begin{aligned}
G & =\{1,2, \cdots, g(n)-1, g(n)\}, \\
H & =\{g(1), g(2), \cdots, g(n-1), g(n)\}, \\
I & =\{g(n+1), g(n+2), g(n+3), \cdots\} .
\end{aligned}
$$

By Claim $1, g(n)-C_{\lambda} \leq g(m)$ for any $m>n$. This implies $\#(G \cap I) \leq C_{\lambda}$. Since $g: \mathbf{Z} \rightarrow \mathbf{Z}$ is bijective, we have

$$
\begin{aligned}
\#(G \backslash(H \cup I)) & =\# G-\#(G \cap H)-\#(G \cap I) \\
& \geq g(n)-n-C_{\lambda}>C_{\lambda}+1 .
\end{aligned}
$$

Thus there exists an integer $j<0$ such that $g(j)>C_{\lambda}+1$. By Claim 1, we have a contradiction;

$$
C_{\lambda} \geq g(j)-g(0)>C_{\lambda}+1 .
$$

Therefore $g(n)-n \leq 2 C_{\lambda}+1$.

The same argument can be applied to $n<0$. We have the claim.

Claim 3. Proposition 4.1 holds.

Proof. Let $n, m \in \mathbf{Z}(n>m)$ and let

$$
A=\left|\frac{f\left(e^{n}\right)-f\left(e^{m}\right)}{e^{n}-e^{m}}\right|=e^{g(n)-n}\left|\frac{1-e^{g(m)-g(n)}}{1-e^{m-n}}\right| .
$$

First, suppose $g(n)>g(m)$. Since $0<e^{g(m)-g(n)}, e^{m-n} \leq e^{-1}$ and by Claim 2, we have

$$
\begin{aligned}
& A \leq e^{2 C_{\lambda}+1} \frac{1}{1-e^{-1}}=\frac{e^{2}}{e-1}(\lambda+1)^{2}, \\
& A \geq e^{-\left(2 C_{\lambda}+1\right)}\left(1-\frac{1}{e}\right)=\left(\frac{e^{2}}{e-1}(\lambda+1)^{2}\right)^{-1} .
\end{aligned}
$$

Next, we suppose $g(n)<g(m)$. Note that, $1 \leq g(m)-g(n) \leq C_{\lambda}$ by Claim 1 . Similarly we have

$$
\begin{aligned}
& A \leq e^{2 C_{\lambda}+1} \frac{e^{C_{\lambda}}-1}{1-e^{-1}}=\frac{e^{2}}{e-1} \lambda(\lambda+1)^{2}, \\
& A \geq e^{-\left(2 C_{\lambda}+1\right)}(e-1)=\left(\frac{e}{e-1}(\lambda+1)^{2}\right)^{-1} .
\end{aligned}
$$

Since $\lambda \geq 1$, we have $1 / M_{\lambda} \leq A \leq M_{\lambda}$.

Corollary 4.2. For a bijection $f: E \rightarrow E, f$ is quasisymmetric if and only if $f$ is biLipschitz. 
Proof. Generally, $M$-biLipschitz mappings are $\eta(t)=M^{2} t$-quasisymmetric. Thus "if" part follows.

Suppose $f$ is $\eta$-quasisymmetric, and let $F(z)=f(z) / f(1)$. Then $F$ is also $\eta$ quasisymmetric with the same $\eta$. Since quasisymmetric mappings take a Cauchy sequence to a Cauchy sequence, we have $F\left(e^{n}\right) \rightarrow 0$ as $n \rightarrow-\infty$. Further $F(1)=1$ and $F$ satisfies the $\lambda:=\eta(1)$-three point condition, see Section 2. Thus, by Proposition 4.1, $F$ is $M_{\lambda}$-biLipschitz. Therefore $f(z)=f(1) F(z)$ is $\max \left\{f(1) M_{\lambda}, M_{\lambda} / f(1)\right\}$ biLipschitz.

Remark 4.3. Contrary to Theorem B, the equivalence in Corollary 4.2 cannot be quantitative: Let $f_{n}: E \rightarrow E, z \mapsto e^{n} z$ for $n \in \mathbf{N}$. Then $f_{n}$ is $\eta(t)=t$-quasisymmetric for any $n \in \mathbf{N}$. However $f_{n}$ is $e^{n}$-biLipschitz, and this biLipschitz constant is sharp.

Finally, we analogously obtain the following theorem.

Theorem 4.4. For a bijection $f: E=\left\{e^{n}\right\}_{n \in \mathbf{Z}} \rightarrow E$, the following conditions are quantitatively equivalent;

1. $f$ is $\eta$-quasisymmetric.

2. $f$ satisfies the $\lambda$-three point condition, and $f\left(e^{n}\right) \rightarrow 0$ as $n \rightarrow-\infty$.

3. $f$ admits a $K$-quasiconformal extension $F: \mathbf{C} \rightarrow \mathbf{C}$.

Proof. $(1) \Rightarrow(2)$ follows for the same reason as in the preceding proof.

Suppose $f$ satisfies the $\lambda$-three point condition and $f\left(e^{n}\right) \rightarrow 0$ as $n \rightarrow-\infty$. Then $g(z)=f(z) / f(1)$ also satisfies the $\lambda$-three point condition and $g\left(e^{n}\right) \rightarrow 0$ as $n \rightarrow-\infty$. Since $g(1)=1$, applying Proposition 4.1, it turns out that $g$ is $M_{\lambda}$-biLipschitz. For the same reason as in the proof of Theorem B, $g$ admits a $K$ quasiconformal extension $G: \mathbf{C} \rightarrow \mathbf{C}$ where $K$ is a constant depending only on $\lambda$. We obtain a $K$-quasiconformal extension $F(z)=f(1) G(z)(z \in \mathbf{C})$ of $f$.

$(3) \Rightarrow(1)$ also follows for the same reason as in the proof of Theorem B.

Remark 4.5. In condition (2), $f\left(e^{n}\right) \rightarrow 0$ as $n \rightarrow-\infty$ is necessary. More precisely, the $\lambda$-three point condition does not imply this property. In fact, $f: E \rightarrow$ $E, e^{n} \mapsto e^{-n}$ satisfies the 1-three point condition, but $f\left(e^{n}\right) \rightarrow \infty$ as $n \rightarrow-\infty$.

\section{Characterization of quasisymmetric images}

In this section, we characterize subsets $E \subset \mathbf{R}$ which are images of some quasisymmetric embeddings $f: \mathbf{Z} \rightarrow \mathbf{R}$. On the other hand, the author have characterized images of quasiconformal mappings as follows;

Theorem 5.1. (F. 2015 [3, Theorem A]) For a subset $E \subset \mathbf{R}$, the following conditions are quantitatively equivalent.

1. There exists a $K$-quasiconformal mapping $F: \mathbf{C} \rightarrow \mathbf{C}$, such that $F(\mathbf{Z})=E$.

2. $E$ can be written as a monotone increasing sequence $E=\left\{a_{n}\right\}_{n \in \mathbf{Z}}$ with $a_{n} \rightarrow \pm \infty$ as $n \rightarrow \pm \infty$, and there exists a constant $M \geq 1$ such that the following inequality holds for all $n \in \mathbf{Z}$ and $k \in \mathbf{N}$;

$$
\frac{1}{M} \leq \frac{a_{n+k}-a_{n}}{a_{n}-a_{n-k}} \leq M
$$

Further, if $E$ satisfies the second condition, there exists a quasiconformal mapping $F: \mathbf{C} \rightarrow \mathbf{C}$ such that $F(n)=a_{n}$ for all $n \in \mathbf{Z}$. 
We will see that the above conditions are the required characterizations. To see this, we can use almost the same proof as [3, Theorem A]. However, we give proofs here for completeness and convenience. First, we prepare some preliminary lemmas.

Remark 5.2. Quasisymmetric mappings take Cauchy sequences to Cauchy sequences. Therefore, if $E \subset \mathbf{R}$ is an image of a quasisymmetric mapping $f: \mathbf{Z} \rightarrow \mathbf{R}$, then $E$ must be closed and discrete in $\mathbf{R}$.

Lemma 5.3. Let $f: \mathbf{Z} \rightarrow \mathbf{R}$ be an $\eta$-quasisymmetric mapping, and let $E:=$ $f(\mathbf{Z})$. Then $\sup E=\infty$ and $\inf E=-\infty$.

Proof. To obtain a contradiction, we assume inf $E>-\infty$. Since $E$ is closed and discrete, we have $\sup E=\infty$. Thus $E$ can be written as a monotone increasing sequence $E=\left\{a_{n}\right\}_{n \in \mathbf{N}}$ with $a_{n} \rightarrow \infty$ as $n \rightarrow \infty$.

Let $g:=f^{-1}: E \rightarrow \mathbf{Z}$. By translation, we may assume $g\left(a_{1}\right)=0$. Further, note that $g$ is $\eta^{\prime}$-quasisymmetric where $\eta^{\prime}(t)=1 / \eta^{-1}(1 / t)$, see [6, Theorem 2.2]. Let $\mu:=\eta^{\prime}(1)$ and consider the set

$$
S:=\left\{\begin{array}{l|l}
k \in \mathbf{N} & g\left(a_{k}\right)=\max _{j=1,2, \cdots, k} g\left(a_{j}\right) \geq \mu
\end{array}\right\} .
$$

Since $g: E \rightarrow \mathbf{Z}$ is bijective, $S$ consists of infinitely many elements. We number $S=\left\{k_{j}\right\}_{j \in \mathbf{N}}$ in ascending order. Then the sequence $\left\{g\left(a_{k_{j}}\right)\right\}_{j \in \mathbf{N}} \subset \mathbf{Z}$ is monotone increasing. On the other hand, there exist infinitely many $n \in \mathbf{N}$ with $g\left(a_{n}\right)<0$. Thus we can find $j, \ell \in \mathbf{N}$ such that $k_{j}<\ell<k_{j+1}$ and $g\left(a_{\ell}\right)<0$. Moreover since $g\left(a_{n}\right) \leq g\left(a_{k_{j}}\right)$ for all $n=1,2, \ldots, k_{j+1}-1$, if $g\left(a_{m}\right)=g\left(a_{k_{j}}\right)+1$ then $m \geq k_{j+1}$. Consequently we confirmed that there exists $k \in S$ and exist $\ell, m \in \mathbf{N}$ such that

1. $k<\ell<m$,

2. $g\left(a_{\ell}\right)<0$ and $g\left(a_{m}\right)=g\left(a_{k}\right)+1$, see Figure 2 .

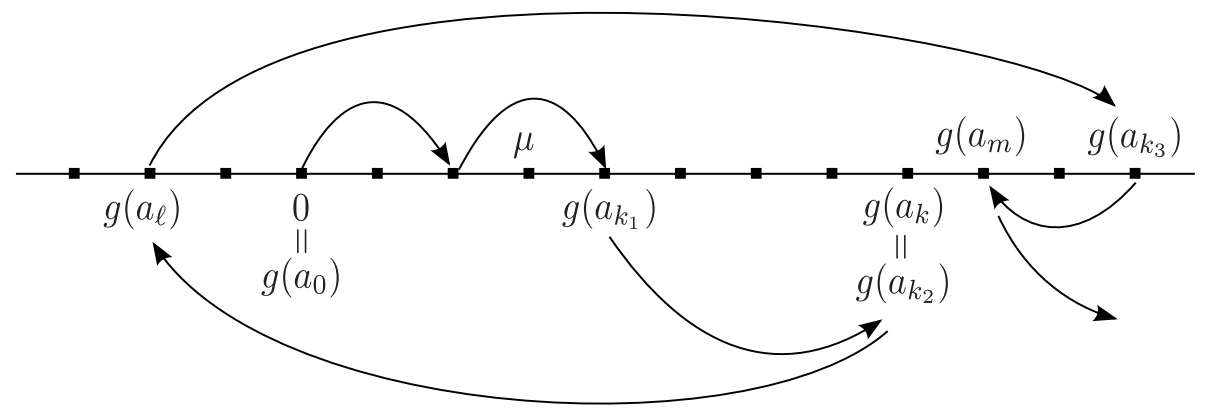

Figure 2.

Therefore, we have a contradiction;

$$
\mu>\eta^{\prime}\left(\left|\frac{a_{k}-a_{\ell}}{a_{k}-a_{m}}\right|\right) \geq\left|\frac{g\left(a_{k}\right)-g\left(a_{\ell}\right)}{g\left(a_{k}\right)-g\left(a_{m}\right)}\right|=g\left(a_{k}\right)-g\left(a_{\ell}\right)>g\left(a_{k}\right) \geq \mu .
$$

Lemma 5.4. Let $E=\left\{a_{n}\right\}_{n \in \mathbf{Z}} \subset \mathbf{R}$ be a monotone increasing sequence with $a_{n} \rightarrow \pm \infty$ as $n \rightarrow \pm \infty$. If $g: E \rightarrow \mathbf{Z}$ is an $\eta^{\prime}$-quasisymmetric bijection, then there exists a constant $L \geq 1$ depending only on $\mu:=\eta^{\prime}(1)$ which satisfies the following inequality for all $n \in \mathbf{Z}$ and $k \in \mathbf{N}$;

$$
\frac{1}{L}<\left|\frac{g\left(a_{n+k}\right)-g\left(a_{n}\right)}{g\left(a_{n}\right)-g\left(a_{n-k}\right)}\right|<L .
$$


Proof. Since $g$ satisfies the $\eta^{\prime}(1)$-three point condition, by Lemma 3.1 we have

$$
\frac{1}{4 \mu^{2}}<\left|\frac{g\left(a_{n+k}\right)-g\left(a_{n}\right)}{g\left(a_{n}\right)-g\left(a_{n-k}\right)}\right|<4 \mu^{2}
$$

where $\mu=\eta^{\prime}(1)$.

Theorem C. For a subset $E \subset \mathbf{R}$, the following conditions are quantitatively equivalent;

1. There exists an $\eta$-quasisymmetric bijection $f: \mathbf{Z} \rightarrow E$.

2. $E$ can be written as a monotone increasing sequence $E=\left\{a_{n}\right\}_{n \in \mathbf{Z}}$ with $a_{n} \rightarrow \pm \infty$ as $n \rightarrow \pm \infty$, and there exists a constant $M \geq 1$ such that the following inequality holds for all $n \in \mathbf{Z}$ and $k \in \mathbf{N}$;

$$
\frac{1}{M} \leq \frac{a_{n+k}-a_{n}}{a_{n}-a_{n-k}} \leq M \text {. }
$$

3. There exists a $K$-quasiconformal mapping $F: \mathbf{C} \rightarrow \mathbf{C}$, such that $F(\mathbf{Z})=E$.

Proof. The equivalence $(2) \Leftrightarrow(3)$ is already confirmed by Theorem 5.1 , see [3, Theorem A]. Further, for the same reason as in the proof of Theorem B, $(3) \Rightarrow(1)$ follows. Thus it suffices to show $(1) \Rightarrow(2)$.

Let us assume that there exists an $\eta$-quasisymmetric bijection $f: \mathbf{Z} \rightarrow E$. By Lemma 5.3, $E$ can be written as a monotone increasing sequence $E=\left\{a_{n}\right\}_{n \in \mathbf{Z}}$ with $a_{n} \rightarrow \pm \infty$ as $n \rightarrow \pm \infty$ (recall $E$ must be closed and discrete in $\mathbf{R}$ ). Let $g:=f^{-1}$. Then $g$ is $\eta^{\prime}$-quasisymmetric where $\eta^{\prime}(t)=1 / \eta^{-1}(1 / t)$. By Lemma 5.4 , there exists a constant $L \geq 1$ depending only on $\eta^{\prime}(1)=1 / \eta^{-1}(1)$ which satisfies the following inequality for any $n \in \mathbf{Z}$ and $k \in \mathbf{N}$;

$$
\frac{1}{L}<\left|\frac{g\left(a_{n+k}\right)-g\left(a_{n}\right)}{g\left(a_{n}\right)-g\left(a_{n-k}\right)}\right|<L .
$$

Therefore we obtain

$$
\left|\frac{a_{n+k}-a_{n}}{a_{n}-a_{n-k}}\right| \leq \eta\left(\left|\frac{g\left(a_{n+k}\right)-g\left(a_{n}\right)}{g\left(a_{n}\right)-g\left(a_{n-k}\right)}\right|\right)<\eta(L) .
$$

and

$$
\left|\frac{a_{n+k}-a_{n}}{a_{n}-a_{n-k}}\right| \geq \eta\left(\left|\frac{g\left(a_{n+k}\right)-g\left(a_{n}\right)}{g\left(a_{n}\right)-g\left(a_{n-k}\right)}\right|^{-1}\right)^{-1}>\frac{1}{\eta(L)} .
$$

\section{Extendability of quasisymmetric embeddings}

We complete this paper, proving the following theorem;

Theorem A. Every $\eta$-quasisymmetric embedding $f: \mathbf{Z} \rightarrow \mathbf{R}$ admits a $K=$ $K(\eta)$-quasiconformal extension $\tilde{f}: \mathbf{C} \rightarrow \mathbf{C}$ where $K(\eta)$ is a constant depending only on $\eta$.

Proof. Let $f: \mathbf{Z} \rightarrow \mathbf{R}$ be an $\eta$-quasisymmetric embedding, and let $E:=f(\mathbf{Z})$. Then, by Theorem $\mathrm{C}$, there exists a $K^{\prime}$-quasiconformal mapping $F: \mathbf{C} \rightarrow \mathbf{C}$ such that $F(\mathbf{Z})=E$, where $K^{\prime}$ depends only on $\eta$. Since compositions of quasisymmetric mappings are also quasisymmetric, $F^{-1} \circ f: \mathbf{Z} \rightarrow \mathbf{Z}$ becomes an $\eta^{\prime}$-quasisymmetric automorphism where $\eta^{\prime}$ depends only on $\eta$. By Theorem B, $F^{-1} \circ f$ admits a $K^{\prime \prime}$ quasiconformal extension $G: \mathbf{C} \rightarrow \mathbf{C}$, where $K^{\prime \prime}$ depends only on $\eta$. Therefore, we 
obtain a $K=K^{\prime} K^{\prime \prime}$-quasiconformal extension $\tilde{f}=F \circ G$ : $\mathbf{C} \rightarrow \mathbf{C}$ of $f$. The proof is completed.

Acknowledgements. I am deeply grateful to Professor Takeo Ohsawa for his guidance and helpful advices. I also would like to thank the referees for careful reading of the manuscript and for many helpful comments and suggestions, which improve significantly the arguments in Section 4. This research is partially supported by Grant-in-Aid for JSPS Fellow 16J02185.

\section{References}

[1] Alestalo, P., and J. VÄisälä: Uniform domains of higher order III. - Ann. Acad. Sci. Fenn. Math. 22:2, 1997, 445-464.

[2] Beurling, A., and L. V. Ahlfors: The boundary correspondence under quasiconformal mappings. - Acta Math. 96, 1956, 125-142.

[3] Fujino, H.: The existence of quasiconformal homeomorphism between planes with countable marked points. - Kodai Math. J. 38:3, 2015, 732-746.

[4] Heinonen, J.: Lectures on analysis on metric spaces. - Universitext, Springer-Verlag, New York, 2001.

[5] Trotsenko, D. A., and J. VÄIsÄLä: Upper sets and quasisymmetric maps. - Ann. Acad. Sci. Fenn. Math. 24:2, 465-488.

[6] Tukia, P., and J. VäIsÄLÄ: Quasisymmetric embeddings of metric spaces. - Ann. Acad. Sci. Fenn. Ser. A I Math. 5:1, 1980, 97-114.

[7] VÄısÄLÄ, J.: Questions on quasiconformal maps in space. - In: Quasiconformal mappings and analysis (Ann Arbor, MI, 1995), Springer, New York, 1998, 369-374.

[8] VelLis, V.: Quasisymmetric extension on the real line. - arXiv:1509.06638, 2015.

Received 28 May 2016• Revised received 12 September $2016 \bullet$ Accepted 7 October 2016 